This item was submitted to Loughborough's Research Repository by the author.

Items in Figshare are protected by copyright, with all rights reserved, unless otherwise indicated.

\title{
Anticipation of aerobic exercise increases planned energy intake for a post- exercise meal
}

PLEASE CITE THE PUBLISHED VERSION

https://doi.org/10.1016/j.appet.2019.03.035

\section{PUBLISHER}

(C) Elsevier BV

\section{VERSION}

AM (Accepted Manuscript)

\section{PUBLISHER STATEMENT}

This paper was accepted for publication in the journal Appetite and the definitive published version is available at https://doi.org/10.1016/j.appet.2019.03.035.

\section{LICENCE}

CC BY-NC-ND 4.0

\section{REPOSITORY RECORD}

Barutcu, Asya, Gemma Witcomb, and Lewis James. 2019. "Anticipation of Aerobic Exercise Increases Planned Energy Intake for a Post-exercise Meal”. figshare. https://hdl.handle.net/2134/37501. 
1 Anticipation of aerobic exercise increases planned energy intake for a post-exercise 2 meal

3

4 Asya Barutcu ${ }^{1}$ Gemma L. Witcomb ${ }^{1}$ and Lewis J. James ${ }^{1}$

51 School of Sport, Exercise and Health Sciences, Loughborough University, 6 Leicestershire, UK, LE11 3TU.

7

\section{Corresponding author}

9 Lewis J. James

10 L.James@lboro.ac.uk

11 School of Sport, Exercise and Health Sciences

12 Loughborough University

13

14

15 Running head: Exercise and meal planning

16

17

18

19

20

21

22

23

24

25

26

27

28

29

30

31

32

33 
34 Key words: Appetite; energy balance; eating behavior; weight loss; physical activity 


\section{Abstract}

In many situations, meals are planned (i.e. what and how much) before they are eaten, but

38 how exercise influences this planning is unknown. Therefore, this study investigated whether

39 anticipation of an exercise session alters food intake planned for post-exercise. Forty (16 40 male) regular exercisers (mean \pm SD; age $23.3 \pm 5.6$ y, BMI $22.7 \pm 3.3 \mathrm{~kg} / \mathrm{m}^{2}$, body fat $25.6 \pm$ $417.6 \%$ ) completed the study. Subjects arrived $\geq 3 \mathrm{~h}$ post-prandial and were given two

42 hypothetical scenarios for the following day: 1) morning rest (REST), or 2) morning rest with 43 the addition of $1 \mathrm{~h}$ of hard aerobic exercise at 10:00-11:00 (EXERCISE). For each scenario 44 subjects had to plan their lunch, to consume at 12:00, by serving themselves cheesy tomato 45 pasta and chocolate buttons. Scenarios were counterbalanced and separated by 5 minutes 46 and foods were not consumed. EXERCISE increased total energy served by $24 \%$ (EXERCISE $473308 \pm 1217 \mathrm{~kJ}$; REST $2663 \pm 924 \mathrm{~kJ}$; $P<0.001$ ), with increases in energy served from both 48 pasta $(+25 \% ; P<0.001)$ and chocolate buttons $(+20 \% ; P=0.024)$. These results suggest 49 aerobic exercise increases planned post-exercise energy intake, if a meal is planned in 50 advance of exercise. Future research should examine the impact of exercise on meal planning 51 at other meals, as well as how this behaviour impacts weight loss with exercise training. 
54 The most recent public health statistics suggest that the prevalence of overweight and obesity continue to rise, with $61 \%$ of UK adults currently classified as overweight or obese (Health Survey for England, 2016). Weight gain occurs due to chronic positive energy balance (i.e. energy intake greater than energy expenditure), leading to accumulation of fat in adipose tissue (Schrauwen, 2007). Increasing physical activity, particularly aerobic activity, is one method of increasing energy expenditure that has been suggested to assist with weight management (Caudwell et al., 2011). The premise of this strategy is that the accumulation of energy expended through physical activity manifests in a negative energy balance and subsequent reduction in body fat levels (Caudwell et al., 2011).

63

64 Clearly, the success of a weight loss strategy involving increased exercise will depend on the degree of compensation through the other components of energy balance (i.e. energy intake, resting energy expenditure and of physical activity; Caudwell et al., 2011). Acute exercise studies have typically reported a transient reduction in subjective appetite (Broom et al., 2007; Pomerleau et al., 2004) and ideal portion size (Farah et al. 2012) during/ after exercise, with minimal effect on subsequent energy intake compared to a resting control trial (Schubert et al., 2013). Whilst some studies report a small increase in absolute energy intake (i.e. total energy consumed) after exercise (Martins et al., 2007a; Martins et al., 2007b; Pomerleau et al., 2004; Shorten et al., 2009), relative energy intake (energy consumed minus energy expended through exercise/ rest) is consistently reduced by exercise. Therefore, acute exercise studies suggest exercise produces an environment conducive to weight loss by increasing energy expenditure without a compensatory increase in energy intake.

However, chronic exercise interventions (i.e. $\geq 8$ weeks) have typically not observed the anticipated weight loss that would be expected given the acute effects of exercise on relative energy intake (King et al., 2008; Turner et al. 2010; Wu et al., 2009). Typically, there is an initial weight loss, however, after this, the rate of weight loss attenuates or weight becomes stable over time (Curioni \& Lourenco, 2005; Wu et al., 2009). Whilst there is likely a reduced energy requirement due to the reduction in body mass over time, these studies also suggest there is some alteration in the other components of energy balance to compensate in some way for the energy expended through exercise training. Given that non-prescribed physical activity energy expenditure (Turner et al., 2010) and resting metabolic rate (Lee et al.. 2009; Speakman \& Selman, 2003) do not appear to change with exercise training, alterations in dietary intake/ eating behaviour have been suggested as the likely cause of this effect (Turner et al. 2010). 
Aside from what we eat, a critical factor is how much we eat. Factors influencing portion size selection strongly affect energy intake, and therefore represent a crucial aspect of energy balance (Brunstrom, 2011). Previous studies that have investigated the relationship between exercise and energy intake have employed an ad-libitum approach to assess energy intake. In this approach, subjects are presented with a variety of food items in excess amounts and are asked to eat or drink until satiated. In day-to-day living this type of eating occasion is relatively rare for most humans, with meals generally involving some planning of the type of foods selected and/ or the amount of food selected, in advance of the eating occasion (Brunstrom, 2011). Additionally, food choice is generally reduced in a laboratory environment. Interestingly, Werle et al. (2011) reported that participants who answered a series of questions related to exercise served themselves more snacks, and therefore more calories, than those in a control group whose questions were unrelated to exercise. In most cases, exercise sessions are scheduled in advance of being undertaken (i.e. individuals know that they will exercise and likely think about the exercise), meaning the size/ nature of any meals prepared/ cooked in advance of exercise might be influenced by the knowledge of the upcoming exercise session. However, the design of most previous exercise studies does not allow any planning behaviour in the context of exercise to be directly evaluated in advance of the session. More recently, Sim et al. (2018) reported that inactive overweight males scoring high for dietary restraint increased energy intake at a snack before exercise. These results suggest that exercise might increase energy intake (or planned energy intake) when decisions are made

111 in advance of exercise, although the training or weight status of the volunteers may have 112 influenced the results.

114 Therefore, the aim of this study was to examine how exercise influences meals planned for 115 the post-exercise period of regularly exercisers by providing subjects with hypothetical exercise and rest scenarios and asking them to plan their post-exercise meal. It was hypothesised that subjects would plan to consume more energy after exercise than after rest. 


\section{Subjects}

121 Twenty-four females (age $21 \pm 3$ years; BMI $22.0 \pm 2.8 \mathrm{~kg} / \mathrm{m}^{2}$; body fat $\% 30.1 \pm 4.5$ ) and

122 sixteen males (age $26 \pm 8$ years; BMI $24.0 \pm 3.7 \mathrm{~kg} / \mathrm{m}^{2}$; body fat $\% 18.3 \pm 5.5$ ) completed this

123 study, which was approved by the Loughborough University Ethics Approvals (Human

124 Participants) Sub Committee (reference number: SSEHS-1917). Before participation, subjects

125 provided written consent, and completed a health screen questionnaire and the Three Factor

126 Eating Questionnaire (TFEQ; Stunkard \& Messick, 1985) which measures the three

127 dimensions (restraint, disinhibition, hunger) of human eating behaviour (See Table 1 for mean

$128 \pm \mathrm{SD}$ ) scores). All subjects were healthy, non-smokers, regular exercisers (3-4 aerobic

129 exercise sessions per week) for at least the previous 6 months, weight stable (body mass

130 within $3 \mathrm{~kg}$ for the past 6 months), not currently dieting and not taking medications known to

131 affect appetite. All subjects were Loughborough University students/staff from a variety of

132 departments. Four of the males and ten of the females scored within the 'clinical range' of the

133 TFEQ (Males: 1 for restraint, 3 for hunger; Females: 4 for disinhibition, 2 for restraint, 1 for

134 hunger; 2 for both restraint and disinhibition, 1 for restraint and hunger), with the 'clinical range'

135 defined as per Stunkard and Messick (1985). Removal of these subjects from the analysis did

136 not alter the results observed and as subjects completed both trials, acting as their own

137 control, these subjects were not removed from the final analysis. Before each visit, subjects

138 refrained from any strenuous exercise or alcohol intake in the preceding 24 hours. Visits were

139 separated by $>3$ days.

140

141 Session 1: Familiarisation session

142 During the first session, the familiarisation trial, subjects visited the laboratory at lunch time

$143(1100-1400 \mathrm{~h})$ at least $3 \mathrm{~h}$ post-prandial to complete pre-trial questionnaires and for the 144 collection of basic anthropometric measurements. Subjects were then familiarised with the 145 meal-planning task to be used in the experimental trial. They were provided with a large bowl 146 of cheese and tomato pasta and a large bowl of chocolate confectionary. They were then 147 asked to serve themselves portions of both foods after being given the following instructions 148 "You are preparing your lunch to eat now. Please serve yourself the amount of food that you 149 would choose to consume to fill you up if you were not going to eat again until you have dinner 150 this evening." Subjects then ate the food they had served to help familiarise themselves with 151 the study foods.

\section{Session 2: Experimental trial}

154 Subjects visited the laboratory at least $3 \mathrm{~h}$ postprandial and performed two hypothetical meal155 planning scenarios; a resting scenario (REST) and an exercising scenario (EXERCISE). The 
order of the scenarios was randomised across subjects in an attempt to control for any order effects and, within subjects, was separated by a 5-minute break. Before each scenario, subjects were asked to rate their subjective appetite using a set of visual analogue scales.

For each scenario, subjects were read the scenario and then asked to "please serve yourself the amount of food that you would choose to consume to fill you up if you were not going to eat again until dinner". For the REST scenario, subjects were told, "For tomorrow, imagine you plan to have your usual breakfast in the morning. You then plan to spend the morning around the house doing some light household activities (e.g. light housework, reading, working on the computer etc.). You plan to have lunch at $\sim 12 \mathrm{pm}$ ". For the EXERCISE scenario, subjects were told, "For tomorrow, imagine you plan to have your usual breakfast in the morning. You then plan to spend most of the morning around the house doing some light household activities (e.g. light housework, reading, working on the computer etc.), except for some time that you will spend exercising. The exercise you plan to do will be 1 hour of hard aerobic exercise from 10 to $11 \mathrm{am}$. You plan to have lunch 1 hour after finishing exercise (i.e. $\sim 12 \mathrm{pm}$ )". In each scenario, subjects were instructed to plan their lunch for the following day in isolation and were provided with the same foods as in the familiarisation trial. Subjects did not consume the foods served in the experimental trial and they were made aware of this beforehand. Food bowls were weighed before and after serving, with manufacturer values used to determine the energy content of meals.

In an attempt to distract subjects from the true focus of the study (i.e. comparison of exercise and rest) they were told that there were multiple hypothetical scenarios involving different types of activities and that they would be randomly assigned to two of these scenarios.

\section{Study Foods}

182 The meal provided was a cheese and tomato pasta as a main course (fusilli pasta, cheddar cheese, Bolognese sauce and olive oil; all Tesco, Cheshunt, UK) and chocolate confectionary as dessert (Cadbury's Dairy Milk Buttons; Cadbury, UK). The cheese and tomato pasta meal was prepared the day prior to the trials using identical cooking and cooling procedures for all meals and was presented to subjects cold. During the familiarisation trial, where subjects ate their selected portion, the pasta was warmed before being eaten. The cheese and tomato pasta was homogenous in nature and provided 6.7 ( $\pm 0.03 \mathrm{SD}$ ) $\mathrm{kJ}^{\circ} \cdot \mathrm{g}^{-1}$ (with $14 \%, 61 \%, 24 \%$ and $2 \%$ of the energy provided by protein, carbohydrate, fat and fibre, respectively). The chocolate confectionary provided $22.2 \mathrm{~kJ} \cdot \mathrm{g}^{-1}$ (with $6 \%, 43 \%, 51 \%$ and $0 \%$ of the energy provided by protein, carbohydrate, fat and fibre, respectively). Foods were presented in large 
192 serving bowls in excess of expected consumption and subjects self-served portions into eating 193 bowls in line with the above instructions.

194

195 Subjective appetite

196 Subjective feelings of hunger, fullness, desire to eat (DTE), and prospective food consumption 197 (PFC) were rated on $100 \mathrm{~mm}$ visual analogue scales. The scales were anchored "not at all/ 198 none at all/ no desire at all" at the $0 \mathrm{~mm}$ point and "extremely/ a lot/ very" at the $100 \mathrm{~mm}$ point. 199 Subjects were instructed to draw a line at the point on the $100 \mathrm{~mm}$ line that corresponded to 200 their appetite.

201

202 Statistical Analysis

203 Data was analysed using SPSS 22.0 for Windows (SPSS Inc., Somers, NY, USA). All data 204 were checked for normality and were analysed using paired t-tests or Wilcoxon signed-rank 205 tests, as appropriate. Statistical significance was accepted at the 5\% level. Results in text and 206 tables are presented as mean \pm SD, unless otherwise stated. Spearman's correlation 207 coefficients were determined for the difference in energy selected between trials (total energy 208 selected in EXERCISE minus total energy selected in REST) and TFEQ scores to establish 209 any relationship. 


\section{Results}

211 Subjective appetite

212 Pre-trial values for hunger $(Z=-1.013 ; P=0.311)$, fullness $(Z=-0.014 ; P=0.989)$, $D T E(Z=-$

213 0.587; $P=0.557)$ and PFC $(Z=-1.356 ; P=0.175)$ were not different between REST and

214 EXERCISE trials (Table 2).

215

216 Portion size selection

217 There was no trial order effect for the total energy content served, with similar amounts served 218 for trial 1 and trial 2 (Trial $12990 \pm 1096 \mathrm{~kJ}$; Trial $22982 \pm 1160 \mathrm{~kJ} ; Z=-0.168 ; P=0.867$ ). 219 Similarly, there was no difference between trial 1 and trial 2 for energy served from pasta $(Z=-$ $2200.511 ; P=0.610)$ or chocolate buttons $(Z=-1.136 ; P=0.256)$. Additionally, there was no 221 interaction effect $(F(1)=0.177 ; P=0.676)$ between condition (EXERCISE and REST) and the 222 order in which trials were completed (i.e. EXERCISE-REST or REST-EXERCISE), suggesting 223 the absence of a contrast/ demand effects.

224

225 During the EXERCISE condition subjects served themselves a significantly larger portion of 226 pasta $(t(39)=-7.343 ; P<0.001 ;$ Figure 1$)$ and chocolate $(Z=-2.251 ; P=0.024 ;$ Figure 1$)$ 227 compared to the REST condition, representing a $24 \%$ increase $(Z=-4.624 ; P<0.001$; Figure 1$)$ 228 in the total energy content of the served food.

When energy served was explored with sex as a between-subject factor (Table 3), responses for energy served for pasta $(F(1)=2.487 ; P=0.100)$ and in total $(F(1)=0.013 ; P=0.908)$ were similar between sexes. Whilst there was a trend for a sex*trial interaction for energy served from chocolate $(F(1)=3.730 ; P=0.061)$, this did not reach statistical significance.

Three Factor Eating Questionnaire (TFEQ)

236 Correlation analyses between the absolute difference in energy selected between trials and three factor eating questionnaire responses revealed weak correlations for disinhibition ( $r=-$ $0.269 ; P=0.094)$ and hunger $(r=0.000 ; P=0.998)$. Although there was a trend for a significant relationship with dietary restraint, the correlation was still weak/moderate $(r=-0.307 ; P=0.054)$. 


\section{Discussion}

248 The aim of the present study was to investigate the effect of exercise on post-exercise meal 249 planning. The main finding was that individuals chose a larger portion size ( $24 \%$ increase in 250 energy content of food served) to consume after a hypothetical future aerobic exercise scenario compared to a hypothetical rest scenario.

To our knowledge, this is the first study to examine the effect of aerobic exercise on meal planning behaviour in anticipation of exercise. Previous investigations have generally taken the approach of performing exercise or rest, with assessment of subsequent appetite and energy intake (Deighton \& Stensel, 2014; Schubert et al., 2013;) after exercise/ rest. Generally, these studies have reported that exercise does not effect subsequent absolute energy intake. Consequently, relative energy intake (energy intake minus energy expended through exercise/rest) is reduced and an acute energy deficit is induced by the exercise bout. In contrast, some studies have observed a reduction (Ueda et al., 2009; Jokisch et al., 2012; Sim et al., 2014) or an increase (Erdmann et al., 2007; Martins et al., 2007a; Shorten et al., 2009) in absolute energy intake after exercise compared to rest. However, these differences in absolute energy intake are generally small and an acute decrease in relative energy intake is consistently observed with exercise.

The results of the present study suggest that knowledge of a future exercise session results in an increase in planned energy intake at a meal after exercise, at least in habitual exercisers. The magnitude of this increase was $\sim 650 \mathrm{~kJ}$ (i.e. $\sim 150 \mathrm{kcal}$ ) at this meal, which is unlikely to fully compensate for energy expended during 1 hour of aerobic exercise. Therefore, these results partially support those of previous studies, as relative energy intake would be expected to be lower in the exercise scenario than the rest scenario. However, it is important to consider that in the present study, only planned energy intake at lunch (i.e. the meal immediately postexercise) was measured. If exercise increases planned energy intake, it is possible this effect might not be constrained to a single meal and that other meals before and/ or after exercise might be subject to the same changes in planning behaviour, providing greater opportunity to compensate for energy expended during exercise. This increase in planned energy intake might attenuate the negative energy balance induced by exercise and consequently might reduce any weight loss with chronic exercise training (Curioni \& Lourenco, 2005). It must also be acknowledged that an hour of 'hard aerobic exercise' would represent different levels of energy expenditure for different subjects, depending on their fitness, mass and exercise of choice. 
283 Whilst previous studies have not assessed meal planning in the context of exercise, Farah et 284 al. (2012) used a computer based method to evaluate the effect of exercise on ideal portion 285 sizes of a variety of different foods. Subjects selected a smaller ideal portion size of a number 286 of different foods (pasta, crackers, KitKat chocolate bar, garlic bread and cheese baguette) 287 after a 60 minute walk compared to a 60 minute resting period. This effect was only apparent immediately after exercise, and coincided with a decrease in subjective hunger at this time. Clearly previous studies that have assessed the effects of exercise on subsequent energy intake will have incorporated some element of meal planning, as subjects will have made decisions about what, and how much food to eat. However, these decisions are likely to have been made after exercise and it is possible the alterations in subjective and physiological mediators of appetite augmented by exercise (Broom et al. 2007) might interact with meal planning behaviour to attenuate energy intake. Therefore, whilst the results of the present study contrast previous findings, they suggest that food intake after exercise might differ based on when decisions about the meal are made (i.e. before or after conducting exercise).

Traditionally, meal size is believed to be regulated by physiological and psychological mechanisms that occur during a meal and lead to the termination of eating. While the disossociation between the two (homeostatic and cognitive) has been argued to be limited (Liu \& Kanoski, 2018), other evidence suggests that meal size might be controlled by decisions made in advance of eating (Guillocheau et al., 2018; Hetherington et al., 2018; Fay et al., 2011; Brunstrom, 2011). In this context, the expected satiety/ satiation of a specific food have been shown to vary widely (Forde et al., 2013; Brunstrom et al., 2008), and are strong predictors of the amount of food served (Brunstrom \& Shakeshaft, 2009; Brunstrom \& Rogers, 2009), with self-served meals tending to be consumed in their entirity (Wansink \& Cheney, 2005). This suggests that there are elements of eating behaviour that are learned based on previous experience of foods/ meals. Indeed, there is evidence to suggest that previous experience of a food modulates expectations about the food's satiation (Brogden \& Almiron-Roig, 2010; Wilkinson \& Brunstrom, 2009) and ultimately these beliefs might play an important role in determining a self-served portion size. Given these learned eating behaviours pertaining to expected satiety/ satiation, it might be plausable to suggest that similar learned responses might govern eating behaviours in response to (or anticipation of) exercise. It is possible that a habitual exerciser might make decisions about meal size and type in response to exercise based on their previous experience of the effect of that exercise session on parameters of appetite/ energy balance regulation. Therefore, this mechanism might act to allow the exerciser to better compensate for the energy expended during exercise and allow them to maintain energy balance. This hypothesis, whilst speculative, is supported by the findings from chronic exercise training studies, that generally report weight loss slows down over time 
320 (Curioni \& Lourenco, 2005). This suggests the possibility of learned compensatory behaviours, 321 as experience with the specific exercise stimulus increases. The subjects recruited for this 322 study were all recreationaly active and regularly undertook aerobic exercise, meaning their 323 previous experiences with the impact of aerobic exercise on appetite/ energy balance etc. 324 might have played a role in their choice to serve more food.

Whilst there was no significant difference in food intake between males and females, there was a trend for selection of the chocolate confectionary to be increase in the exercise trial in females, but not males. This trend is interesting and should be explored in future studies that are better powered to investigate sex-specific differences A recent study found that palatable foods may be more rewarding to females than males (Sinclair et al., 2017). The underlying cause for this behaviour is thought to be the increased responsiveness of neural substrates that settle the hedonic and motivational responses to palatable foods in females (Stoeckel et al., 2008). It has also been found that in western countries like the Unites States, Canada, Spain and United Kingdom chocolate is the single most desired food among women, and recurring changes in cravings have been reported to differ between different phases of the menstrual cycle (Asarian \& Geary, 2013). Although female subjects of this study were tested at an uncontrolled point in their menstrual cycle, both exercise and rest scenarios were completed on the same day, thus controlling for any hormonal-based effects.

340 Typically, studies that have investigated the effects of exercise on appetite, exclude subjects 341 who score within the clinical range of the Three Factor Eating Questionnaire. In an attempt to examine whether elevated scores were driving the observed effects, correlation analyses were carried out. However, these revealed only weak/ moderate associations and removal of these subjects did not change the reported results. There was a trend for a weak/ moderate negative relationship between dietary restraint sore and additional energy consumed in the EXERCISE trial, suggesting that those with greater restraint are less likely to compensate for energy expended through exercise by increased mealplanning behavior. Although this relationship was pretty weak, it is interesting that Sim et al (2018) reported the reverse effect, in that overweight/ obese individuals with high dietary restraint were more likely to increase energy intake at a pre-exercise snack meal. These differential findings might be explained by differences in the activity or weight status of the voluneteers (i.e. lean regular exercisers in the present study vs overweight/obese sedentary individuals in the study of Sim et al. [2018]). Estimation of energy expended during exercise is achieved with varying success for lean individuals (Holliday \& Blannin, 2014; Willbond et al., 2010), but this ability appears to be worse in overweight/ obese individuals (Brown et al., 2016), perhaps explaining the difference in findings between the present study and that of Sim et al. (2018). Future studies should 
357 better consider the interaction between restraint and possibly disinhibition and meal planning 358 responses in the context of exercise. Additionally, weight status should also be investigated in 359 this regard.

360

361 In conclusion, this study demonstrates that knowledge of a planned aerobic exercise session 362 increases portion size selection by $\sim 24 \%$. This finding suggests that aerobic exercise might 363 impact meal planning, at least in regular exercisers, which might account for some of the 364 reasons behind stabilisation of weight loss in chronic exercise intervention studies. Future 365 studies should examine meal planning in response to both acute and chronic aerobic exercise, 366 as well as other exercise modalities. 
Asarian, L., \& Geary, N. (2013) "Sex differences in the physiology of eating", American Journal of Physiology. Regulatory, Integrative and Comparative Physiology, 305, 1215-1267.

Brogden, N., \& Almiron-Roig, E. (2010). Food liking, familiarity and expected satiation selectively influence portion size estimation of snacks and caloric beverages in men. Appetite, 55(3), 551555.

Broom, D. R., Stensel, D. J., Bishop, N. C., Burns, S. F., \& Miyashita, M. (2007) "Exercise-induced suppression of acylated ghrelin in humans", Journal of Applied Physiology, 102, 2165-2171.

Brown, R. E., Canning, K. L., Fung, M., Jiandani, D., Riddell, M. C., Macpherson, A. K., \& Kuk, J. L. (2016) Calorie Estimation in Adults Differing in Body Weight Class and Weight Loss Status. Medicine and Science in Sports and Exercise, 48, 521-526.

Brunstrom, J. M., \& Shakeshaft, N. G. (2009) "Measuring affective (liking) and non-affective (expected satiety) determinants of portion size and food reward", Appetite, 52, 108-114.

Brunstrom, J. M., Shakeshaft, N. G., \& Scott-Samuel, N. E. (2008) "Measuring 'expected satiety' in a range of common foods using a method of constant stimuli", Appetite, 51, 604-614.

Brunstrom, J. M. (2011) "Session 1: Balancing intake and output: food v. exercise. The control of meal size in human subjects: a role for expected satiety, expected satiation and pre-meal planning", Proceedings of the Nutrition Society, 70, 155-161.

Caudwell, P., Gibbons, C., Hopkins, M., Naslund, E., King, N., Finlayson, G., \& Blundell, J. (2011) “The influence of physical activity on appetite control: an experimental system to understand the relationship between exercise-induced energy expenditure and energy intake", Proceedings of the Nutrition Society, 70, 171-180.

Curioni, C. C., \& Lourenco, P. M. (2005) "Long-term weight loss after diet and exercise: a systematic review", International Journal of Obesity, 29, 1168-1174.

Deighton, K., \& Stensel, D. (2014) "Creating an acute energy deficit without stimulating compensatory increases in appetite: is there an optimal exercise protocol?" Proceedings of the Nutrition Society, 73, 352-358.

Erdmann, J., Tahbaz, R., Lippl, F., Wagenpfeil, S., \& Schusdziarra, V. (2007) "Plasma ghrelin levels during exercise - Effects of intensity and duration", Regulatory Peptides, 143, 127-135.

Forde, C. G., Van Kuijk, N., Thaler, T., De Graaf, C., \& Martin, N. (2013). Oral processing characteristics of solid savoury meal components, and relationship with food composition, sensory attributes and expected satiation. Appetite, 60, 208-219.

Fay, S. H., Ferriday, D., Hinton, E. C., Shakeshaft, N. G., Rogers, P. J., \& Brunstrom, J. M. (2011). What determines real-world meal size? Evidence for pre-meal planning. Appetite, 56(2), 284-289.

Guillocheau, E., Davidenko, O., Marsset-Baglieri, A., Darcel, N., Gaudichon, C., Tomé, D., \& Fromentin, G. (2018). Expected satiation alone does not predict actual intake of desserts. Appetite, 123, 183-190.

Health Survey for England 2016: Adult overweight and obesity - published in 13 December 2017. https://digital.nhs.uk/catalogue/PUB30169 Accessed on 06/02/2018. 
Hetherington, M. M., Blundell-Birtill, P., Caton, S. J., Cecil, J. E., Evans, C. E., Rolls, B. J., \& Tang, T. (2018). Understanding the science of portion control and the art of downsizing. Proceedings of the Nutrition Society, 1-9.

Holliday, A., \& Blannin, A. K. (2014) Matching energy intake to expenditure of isocaloric exercise at high- and moderate-intensities. Physiology and Behavior, 10, 120-126.

Liu, C. M., \& Kanoski, S. E. (2018). Homeostatic and non-homeostatic controls of feeding behavior: Distinct vs. common neural systems. Physiology \& Behavior.

Jokisch, E., Coletta, A. \& Raynor, H.A. (2012) “Acute energy compensation and macronutrient intake following exercise in active and inactive males who are normal weight", Appetite, 58, 722-729.

King. N. A., Hopkins, M., Caudwell, P., Stubbs, R. J., \& Blundell, J. E. (2008) "Individual variability following 12 weeks of supervised exercise. Identification and characterization of compensation for exercise-induced weight loss", International Journal of Obesity, 32, 177-184.

Martins, C., Morgan, L. M., Bloom, S. R., \& Robertson, M. D. (2007a) "Effects of exercise on gut peptides, energy intake and appetite", Journal of Endocrinology, 193, 251-258.

Martins., C., Truby, H., \& Morgan, L.M. (2007b) "Short-term appetite control response to a 6-week exercise programme in sedentary volunteers", British Journal of Nutrition, 98, 834-842.

Niblett, Paul (2017) "Statistics on Obesity, Physical Activity and Diet", NHS National Statistics, https://www.gov.uk/government/uploads/system/uploads/attachment data/file/613532/obesphys-acti-diet-eng-2017-rep.pdf.

Pomerleau, M., Imbeault, P., Parker, T., \& Doucet, E. (2004) "Effects of exercise intensity on food intake and appetite in women", American Journal of Clinical Nutrition, 80, 1230-1236.

Schrauwen, P. (2007) "High-fat diet, muscular lipotoxicity and insulin resistance", Proceedings of the Nutrition Society, 66, 33-41.

Schubert, M. M., \& Desbrow, B. \& Sabapathy, S. \& Leveritt, M. (2013) "Acute exercise and subsequent energy intake. A meta-analysis", Appetite, 63, 92-104.

Sim, A., Wallman, K. E., Fairchild, T. J., \& Guelfi, K. J. (2014) "High-intensity intermittent exercise attenuates ad-libitum energy intake", International Journal of Obesity, 38, 417-422.

Sinclair, E. B., \& Hildebrandt, B. A. \& Culbert, K. M. \& Klump, K. L. \& Sisk, C. L. (2017) "Preliminary evidence of sex differences in behavioural and neural responses to palatable food reward in rats", Physiology and Behaviour, 176, 165-173.

Shorten, A. L., Wallman, K. E., \& Guelfi, K. J. (2009) “Acute effect of environmental temperature during exercise on subsequent energy intake in active men", American Journal of Clinical Nutrition, 90, 1215-1221.

Stoeckel, L. E., Weller, R. E., Cook III, E. W., Twieg, D. B., Knowlton, R. C., \& Cox, J. E. (2008) "Widespread reward-system activation in obese women in response to pictures of high-calorie foods", Neurolmage, 41, 636-647.

Stunkard, A., \& Messick, S. (1985) "The three-factor eating questionnaire to measure dietary restraint, disinhibition and hunger", Journal of Psychosomatic Research, 29, 71-83. 
445 Ueda, S., Yoshikawa, T., Katsura, Y., Usui, T., \& Fujimoto, S. (2009) "Comparable effects of moderate intensity exercise on changes in anorectic gut hormone levels and energy intake to high intensity exercise", Journal of Endocrinology, 203, 357-364.

448 Werle, C. O. C., Wansink, B., \& Payne, C. R. (2011) "Just thinking about exercise makes me serve more food. Physical activity and calorie compensation", Appetite, 56, 332-335.

450 Wansink, B., \& Cheney, M. M. (2005) "Super bowls: serving bowl size and food consumption", JAMA, 293, 1727-1728.

452 Willbond, S. M., Laviolette, M. A., Duval, K., \& Doucet, E. (2010) Normal weight men and women overestimate exercise energy expenditure. Journal of Sports Medicine and Physical Fitness, 50, 377-384.

455 Wu, T., Gao, X., Chen, M., \& van Dam, R. M. (2009) "Long-term effectiveness of diet-plusexercise interventions vs. diet-only interventions for weight loss: a meta-analysis", Obesity Reviews, 10, 313-323. 
459 Table 1. Three Factor Eating Questionnaire scores for males $(n=16)$ and females $(n=24)$.

460 Values are mean \pm SD.

461

\begin{tabular}{lccc}
\hline & Male & Female & Total \\
\hline Restraint & $9 \pm 4$ & $11 \pm 4$ & $10 \pm 4$ \\
Disinhibition & $5 \pm 2$ & $8 \pm 4$ & $7 \pm 4$ \\
Hunger & $6 \pm 3$ & $6 \pm 3$ & $6 \pm 3$ \\
\hline
\end{tabular}

462

463

464

465

466

467

468

469

470

471

472

473

474

475

476

477

478

479

480

481

482

483

484

485

486

487

488

489

490

491

492

493

494

495

496

497

498

499

500

501

502 
503 Table 2. Pre-trial subjective appetite ratings $(0-10 \mathrm{~cm})$. Values are mean \pm SD.

504

\begin{tabular}{lcc}
\hline & REST & EXERCISE \\
\hline Hunger $(\mathbf{0}-\mathbf{1 0} \mathbf{~ c m})$ & $6.9 \pm 2.1$ & $7.0 \pm 1.8$ \\
Fullness $(\mathbf{0}-\mathbf{1 0} \mathbf{~ c m})$ & $2.3 \pm 2.1$ & $2.2 \pm 1.5$ \\
DTE $(\mathbf{0}-10 \mathbf{~ c m})$ & $7.1 \pm 2.2$ & $7.2 \pm 1.9$ \\
PFC $(\mathbf{0}-10 \mathbf{c m})$ & $6.8 \pm 1.9$ & $6.8 \pm 1.9$ \\
\hline
\end{tabular}

505

506

507

508

509

510

511

512

513

514

515

516

517

518

519

520

521

522

523

524

525

526

527

528

529

530

531

532

533

534

535

536

537

538

539

540

541

542

543

544

545 
546 Table 3. Energy (kJ) selected for pasta, chocolate and total in males $(n=16)$ and females ( $n$ $547=24)$ during EX and REST trials. Values are mean \pm SD.

548

\begin{tabular}{ccccccc}
\hline & \multicolumn{2}{c}{ Pasta } & \multicolumn{2}{c}{ Chocolate } & \multicolumn{2}{c}{ Total } \\
& Male & Female & Male & Female & Male & Female \\
\hline & & & & & & \\
EXERCISE & $3169 \pm$ & $2189 \pm$ & $699 \pm$ & $746 \pm$ & $3868 \pm$ & $2935 \pm$ \\
& 967 & 615 & 750 & 534 & 1427 & 905 \\
& & & & & & \\
REST & $2501 \pm$ & $1762 \pm$ & $736 \pm$ & $519 \pm$ & $3237 \pm$ & $2280 \pm$ \\
& 691 & 483 & 426 & 436 & 956 & 684 \\
\hline
\end{tabular}

549

550

551

552

553

554

555

556

557

558

559

560

561

562

563

564

565

566

567

568

569 


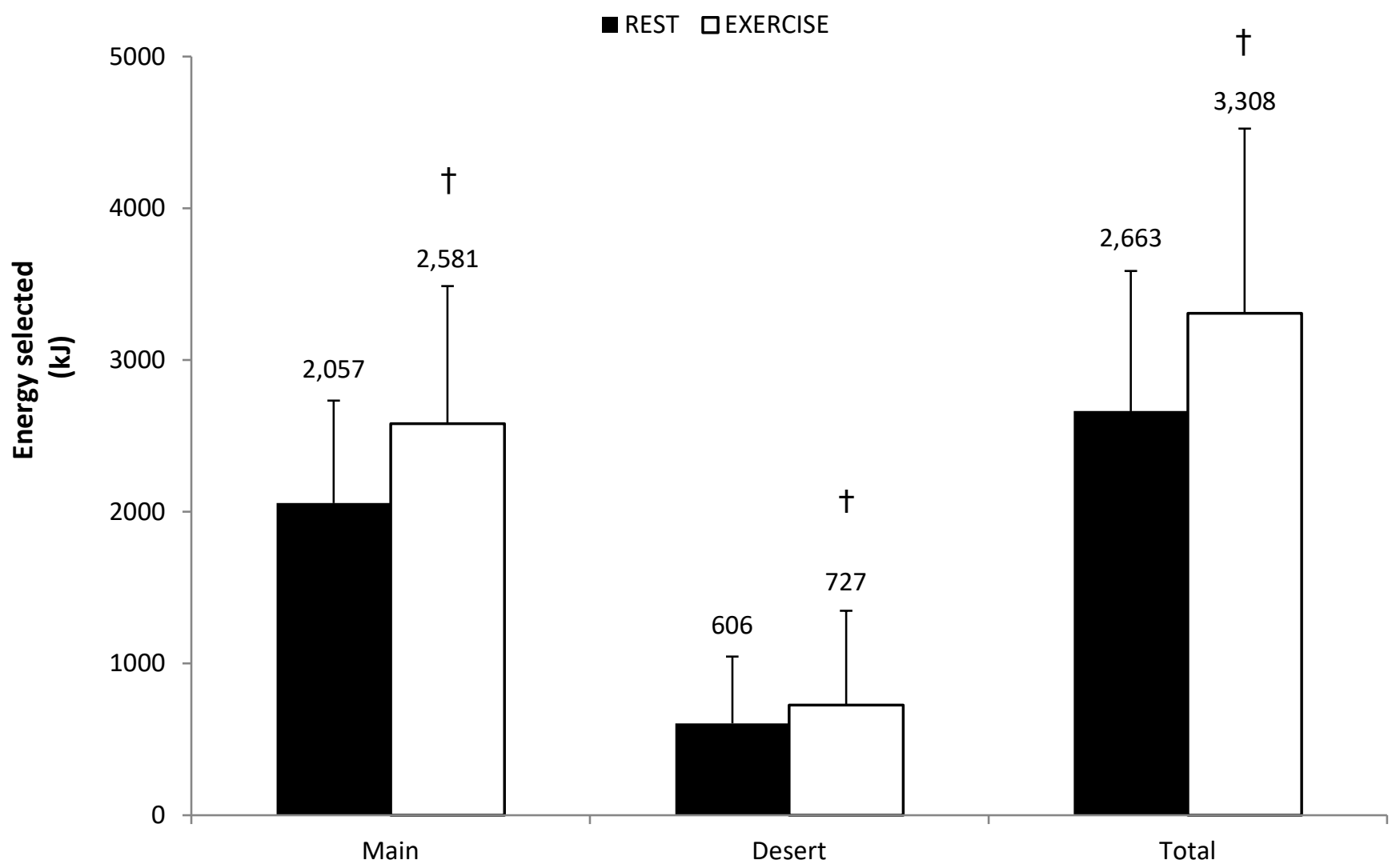

570

571 Figure 1. Energy (kJ) selected in REST (dark bar) and EXERCISE (white bar) conditions.

572 Values are mean \pm SD $(n=40)$. ${ }^{\dagger}$ Significantly different between trials $(P<0.05)$.

573

574

575

576

577

578

579

580

581

582

583

584

585

586

587 